\title{
VASCULAR RESPONSES TO THE SUBCUTANEOUS INJECTION OF GOLD NANOPARTICLES
}

\section{A.E. Volkov', G.V. Reva ${ }^{1,2}$, M.V. Slesarenko', Y.V. Gordzievskaya' , V.S. Tudakov' ${ }^{3}$ E.V. Tsegolnik', I.V. Reva ${ }^{1,2}$}

\author{
${ }^{1}$ Far Eastern Federal University, Vladivostok, Russia \\ (690950, Vladivostok, Suchanova street,8); e-mail: eug_alp@mail.ru \\ ${ }^{2}$ International Medical Research Center (IMERC), Niigata, Japan, \\ e-mail:avers2@yandex.ru; \\ ${ }^{3}$ Pacific State Medical University, Vladivostok, Russia, Ostryakova \\ street, 2; e-mail: vl.tamara.n@yandex.ru
}

This work was supported by Science Foundation Far Eastern Federal University, in the framework of the state task from 17.5740/2017/6.7.

\begin{abstract}
A B STRACT - The study presents the results of a study of the inhibitory role of gold nanoparticles, administered subcutaneously to mice line CBA. The migration paths of gold nanoparticles after subcutaneously injection have been studied, and the mechanisms of their influence on the tissue surrounding the injection site of nanoparticles have been examined. After subcutaneous injection, gold nanoparticles have been found to undergo phagocytosis by macrophages, some of which migrate to lymph node lymphoid follicles, and some enter the lumen of blood vessels, where nanoparticles exit the macrophage cytoplasm and enter the bloodstream. It has been established that as a result of the toxic effect of macrophages loaded with nanoparticles, the endothelium of the vessels growing into the tumor is destroyed. It was concluded that inhibition of angiogenesis and death of blood vessels in the tissues after the introduction of nanoparticles occurs in two ways. The first is not associated with direct inhibition of endothelial growth factor (VEGF), but with the deactivation of macrophages that produce VEGF, the molecules of which stimulate the formation of endothelium in growing blood vessels; and the second mechanism is implemented through the direct death of the endothelium during the migration of the macrophage through the vessel wall.
\end{abstract}

KEY W ORDS - gold nanoparticles, toxicokinetics, mechanism of migration of nanoparticles of gold when a skin.

There is an assumption that gold nanoparticles can be used in oncology for treating tumors. [4] After the introduction of gold nanoparticles and exposure to infrared rays, there is a local death of cancer cells, but there is no data on the generalized effect and side effects of gold nanoparticles on experimental animals and humans, which dictates the need for research in this direction.
Gold nanoparticles with a number of unique characteristics (optical properties, strength, high surface area) are mainly used for diagnostic purposes $[1,8]$. Gold nanoparticles can serve to amplify the signal from cancer cells in diagnosing a tumor by binding nanoparticles with antibodies to tumor cells [9]. Wang et al (2011) used gold nanoparticles to increase the sensitivity of cancer cells to drugs [15]. An electrochemical approach based on partial replacement of electrodes with gold nanoparticles has recently been used for tagless detection of a cancer-embryonic antigen.

The attachment of oligonucleotide sequences complementary to the target DNA molecule to the surface of gold nanoparticles for the colorimetric detection of DNA is a relatively new diagnostic technique, presenting an alternative to fluorescent and radioimmune methods [7]. This technique can also be used for the detection of extremely low (atom-molar) concentrations of protein in biological media. Thus, this technique was applied to measure the concentration of $\beta$-amyloid fragments (the putative marker of Alzheimer's disease) present in the cerebrospinal fluid in negligible amounts [6].

At the present stage, it has been established that gold nanoparticles are capable of destroying blood vessels of a cancerous tumor; on the basis of this, it has been suggested that the mechanism of vascular damage is associated with inhibition of endothelial growth factor (VEGF) [14, 10,3].

It is known that gold preparations exhibit an antibacterial effect, for example, against Helicobacter pylori, toxoplasma, and also exhibit antifungal activity. At the same time they give a pronounced side effect.

Many cancer cells have a protein all over their surface, known as epidermal growth factor receptor (EFGR), while healthy cells usually do not express this protein so strongly. Conjugation (or binding) of gold nanoparticles with anti-EFGR antibodies, commonly called anti-EFGR, allowed the researchers to bind the nanoparticles to the cancer cells themselves. Therefore, the properties of NPG are studied as radio modifiers for use as targets for tumor damage. Also currently, work is underway to create complexes of gold nanoparticles and antibiotics for the destruction of antibiotic-resistant microorganisms [12, 18]. Further improvement of diagnostic approaches based on the use of gold nanoparticles is associated with the devel- 
opment of methods for functionalizing the surface of these nanoparticles using carbohydrates and increasing the sensitivity of nanosensors based on gold nanoparticles with the profiling of their diagnostic capabilities.

Despite numerous studies on the possibility of using gold nanoparticles in the development of new antibiotics, their use is not yet possible due to the presence of side effects and the lack of data on their generalized effect on not only humans, but also experimental animals $[5,13,2,17]$. The limited use of nanoparticles is due to the fact that gold compounds are toxic, accumulate in the kidneys, liver, spleen and hypothalamus, which can lead to organic diseases and dermatitis, stomatitis and thrombocytopenia $[16,11]$.

Therefore, the urgency of the need to study the toxicokinetics of gold nanoparticles on the material of experimental animals for further extrapolation to the human body is rapidly increasing.

\section{MATERIAL AND METHODS}

The study was carried out on 35 male CBA mice. Group 1 intact controls $(n=5)$ were kept together with other groups under similar conditions (temperature, humidity, day/night, and nutrition). Control group $2(\mathrm{n}=5)$ mice were subcutaneously injected with 0.5 $\mathrm{ml}$ isotonic saline. Experimental group $3(\mathrm{n}=25)$ mice were subcutaneously injected with $0.5 \mathrm{ml}$ gold NP suspension ( $50 \%$ dilution in isotonic saline). Sequentially, after 1,2, 3, 4, 5 days, the rats were killed, soft tissues of the proximal third of the posterior thigh were isolated, including lymphoid tissue of the inguinal region, and then paraffin blocks were made using the classical method. The resulting sections were dewaxed, and then stained with standard hematoxylin-eosin. An illustrative material was obtained using an Olympus Bx51 microscope with a digital camera CDx25, and then analyzed using original Olympus morphometric computer programs.

Gold nanoparticles in a colloidal solution had sizes of 10-20 nm and were obtained at the Institute of Chemistry of the Far Eastern Branch of the Russian Academy of Sciences (Vladivostok).

\section{RESULTS}

No morphological changes were found in the tissues of mice from both control groups: typical structure of the connective tissue, vascular walls, muscle tissue, skin, and inguinal lymph nodes of the hip were found during all periods of the study.

The material obtained on the first day of the experiment from rats that received subcutaneous injections of gold nanoparticles made it possible to establish the effects of pronounced perivascular leukocyte infiltration around the wall of blood vessels near the contamination of nanoparticles in the tissue. On the second, third and all subsequent days of the experiment, blood vessels of different caliber are detected, in which hypertrophy and death of endotheliocytes, destruction of the basement membrane are identified. The cytoplasm of endotheliocytes bulges into the lumen of the endothelium, and on the side of the vessel adjacent to the zone of introduction of nanoparticles. On the $1^{\text {st }}$ day, gold nanoparticles are identified to a greater degree in the free state, and then the surrounding white blood cells contaminate them gradually. The number of nanoparticles in the cytoplasm of macrophages from single ones in the first-second day after injections increases by the $3^{\text {rd }}$ day until the cytoplasm is full of nanoparticles. On the 3rd day, both free nanoparticles and contaminated and filled the entire cytoplasm of leukocytes are identified.

We noted that macrophages release lysosomes, as well as leukocyte migration in the direction of blood vessels, into the surrounding tissues which the nanoparticles were introduced. At 4-5 days, the main mass of macrophages is identified only near the wall of blood vessels. Also in the vessels, the death of a part of macrophages is observed with the release of gold nanoparticles into the blood. The second way to exit nanoparticles in the lumen of blood vessels is degranulation.

We noted that a part of macrophages loaded with gold nanoparticles migrates to T-dependent zones of lymphoid follicles, as we think, for the antigen presentation of nanoparticles and the activation of the T-lymphocyte pool in the direction of blast transformation of immune cell differenon for the implementation of cellular immune responses.

Thus, the dynamics of the migration of gold nanoparticles, introduced subcutaneously to rats, has the following algorithm:

The $1^{\text {st }}$ day after the administration is accompanied by phagocytosis of gold nanoparticles in the interstitial tissue, as we assume, by two types of macrophages: monocytic and T-lymphocytic. Monocytic phagocytes carry out the delivery of gold nanoparticles into the lumen of blood vessels, releasing them and partially releasing them into the blood due to the death and rupture of the cell membrane; T lymphocytic cells deliver gold nanoparticles to the lymphoid tissue of rat lymph nodes for antigen presentation and induction of blast transformation of the effector cells of transplantation immunity.

On the second day, the number of phagocytosed nanoparticles in macrophages increases, the number of phagocytes increases, which migrate in the direction of the blood vessels. The endothelium of the vascular wall is hypertrophied. Moreover, hypertrophy does 
not develop over the entire surface of the inner wall of blood vessels, but only in the zone adjacent to the site of introduction of gold nanoparticles, the result of hypertrophy is the death of endotheliocytes. On days 2 and 3 , migration of macrophages in 2 directions is identified: to the blood vessels and in the direction to the lymphoid tissue to the periphery of the lymphoid follicle to T-dependent zones for antigen presentation. On the $4^{\text {th }}$ day, macrophages migrating into the vessel lumen are determined, the cytoplasm of which is filled with gold nanoparticles. We have noted that there are two mechanisms for the removal of gold nanoparticles into the vessel lumen: one group of macrophages releases gold nanoparticles as a result of degranulation, the second due to death.

The obtained data on the dynamics of the migration of gold nanoparticles are important for the development of diagnostic and therapeutic measures. It is necessary to implement diagnostic and therapeutic measures in treating tumors on the first day after local subcutaneous administration of gold nanoparticles, since on the second day most of them undergo phagocytosis and migrate in different directions from the site of administration, therefore, the effectiveness of therapeutic measures in the later periods will be reduced.

Thus, we obtained data confirming the ability of effector cells of the phagocytic link loaded with gold nanoparticles to destroy the vascular wall, leading to an increase in its permeability due to the death of the endothelium. We suggest that the mechanism for reducing the growth factor of the endothelium and the absence of angiogenesis in the tumor is associated with the deactivation of macrophages that produce VEGF due to the phagocytosis of a large number of nanoparticles.

\section{REFEREN CES}

1. Arami H, Patel CB, Madsen SJ, Dickinson PJ, Davis RM, Zeng Y, STurges BK, Woolard KD, HabTe FG, Akin D, Sinclair R, Gambhir SS. Nanomedicine for Spontaneous Brain Tumors: A Companion Clinical Trial. ACS Nano. 2019 Feb 4.

2. Arya M, Mishra N, Singh P, Tripathi CB, Parashar P, Singh M, Gupta KP, Saraf SA. In vitro and in silico molecular interaction of multiphase nanoparticles containing inositol hexaphosphate and jacalin: Therapeutic potential against colon cancer cells (HCT-15). J Cell Physiol. 2019 Jan 29.

3. Chen YW, Hsieh SC, Yang YC, Hsu SH, Kung ML, Lin PY, HSIEH HH, Lin CH, TANG CM, HuNG HS. Functional engineered mesenchymal stem cells with fibronectin-gold composite coated catheters for vascular tissue regeneration. Nanomedicine. 2018 Apr;14(3):699-711.
4. Cole LE, McGinnity TL, Irimata LE, VargoGOGOLA T, ROEDER RK. Effects of bisphosphonate ligands and PEGylation on targeted delivery of gold nanoparticles for contrast-enhanced radiographic detection of breast microcalcifications. Acta Biomater. 2018 Dec;82:122-132.

5. Fadel M, Kassab K, Youssef T, El-Kholy AI. One-step synthesis of Phyto-polymer coated gold nanospheres as a delivery system to enhance resveratrol cytotoxicity. Drug Dev Ind Pharm. 2019 Feb 8:1-29.

6. Hua X, Zhou X, Guo S, Zheng T, Yuan R, Xu W. Determination of Alzheimer biomarker DNA by using an electrode modified with in-situ precipitated molybdophosphate catalyzed by alkaline phosphataseencapsulated DNA hydrogel and target recycling amplification. Mikrochim Acta. 2019 Feb 4;186(3):158.

7. Li M, Lao YH, Mintz RL, Chen Z, Shao D, Hu H, Wang HX, Tao Y, LeOng KW. A multifunctional mesoporous silica-gold nanocluster hybrid platform for selective breast cancer cell detection using a catalytic amplification-based colorimetric assay. Nanoscale. 2019 Feb 7;11(6):2631-2636.

8. Mahato K, Nagpal $S$, Shah MA, Srivastava A, Maurya PK, Roy S, Jaiswal A, Singh R, Chandra P. Gold nanoparticle surface engineering strategies and their applications in biomedicine and diagnostics. 3 Biotech. 2019 Feb;9(2):57.

9. Nima ZA, Watanabe F, Jamshidi-Parsian A, Sarimollaoglu M, Nedosekin DA, Han M, Watts JA, Biris AS, Zharov VP, Galanzha EI. Bioinspired magnetic nanoparticles as multimodal photoacoustic, photothermal and photomechanical contrast agents. Sci Rep. 2019 Jan 29;9(1):887.

10. Satapathy SR, Nayak A, Siddharth $S$, Das $S$, Nayak D, Kundu CN. Metallic gold and bioactive quinacrine hybrid nanoparticles inhibit oral cancer stem cell and angiogenesis by deregulating inflammatory cytokines in $\mathrm{p} 53$ dependent manner. Nanomedicine. 2018 Apr;14(3):883-896.

11. Sztandera K, Gorzkiewicz M, KlajnertMaculewicz B. Gold Nanoparticles in Cancer Treatment. Mol Pharm. 2018 Nov 30.

12. Terán-Navarro H, Calderon-Gonzalez R, SAlcines-Cuevas D, García I, Marradi M, Freire J, Salmon E, Portillo-Gonzalez M, Frande-Cabanes E, García-Castaño A, Martinez-Callejo V, Gomez-Roman J, Tobes R, Rivera F, Yañez-Diaz S, Álvarez-DomínguEz C. Pre-clinical development of Listeria-based nanovaccines as immunotherapies for solid tumours: insights from melanoma. Oncoimmunology. 2018 Nov 10;8(2):e1541534.

13. van Pomeren M, Peijnenburg WJGM, Vlieg RC, van Noort SJT, Vijver MG. The biodistribution and immuno-responses of differently shaped non-modified gold particles in zebrafish embryos. Nanotoxicology. 2019 Feb 4:1-14.

14. Wagner O, Schultz M, Edri E, Meir R, Barnoy E, Meiri A, Shpaisman H, Sloutskin E, 
ZALEVSKY Z. Imaging of nanoparticle dynamics in live and apoptotic cells using temporally-modulated polarization. Sci Rep. 2019 Feb 7; 9 (1):1650.

15. Wang F., Wang Y.C., Dou S., XiOng M.H., Sun T.M., WANG J. Doxorubicin-tethered responsive gold nanoparticles facilitate intracellular drug delivery for overcoming multidrug resistance in cancer cells // ACS Nano. 2011 May 24; 5 (5): 3679-92.

16. Xu M, Soliman MG, Sun X, Pelaz B, Feliu N, Parak WJ, Liu S. How Entanglement of Different Physicochemical Properties Complicates the Prediction of in Vitro and in Vivo Interactions of Gold Nanoparticles. ACS Nano. 2018 Oct 23;12(10): 10104-10113.
17. Yang P, Pageni P, Rahman MA, Bam M, Zhu T, Chen YP, Nagarkatti M, Decho AW, Tang C. Gold Nanoparticles with Antibiotic-Metallopolymers toward Broad-Spectrum Antibacterial Effects. Adv Healthc Mater. 2018 Nov 27:e1800854.

18. Yang X, Wei Q, Shao H, Jiang X. Multivalent aminosaccharide-based gold nanoparticles as narrowspectrum antibiotics in vivo. ACS Appl Mater Interfaces. 2019 Feb 4. 